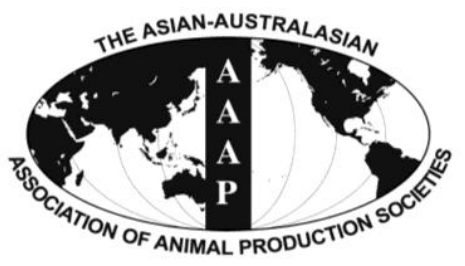

Asian-Aust. J. Anim. Sci.

Vol. 25, No. 6 : 785 - 788

June 2012

www.ajas.info

http://dx.doi.org/10.5713/ajas.2011.11390

\title{
Effect of Peripheral Administration of Kisspeptin-10 on Dynamic LH Secretion in Prepubertal Ewes
}

\author{
Jun Wang ${ }^{1,2}$, Lei Sun ${ }^{2}$, Tao Zhang ${ }^{1,3}$, Haizhu Zhou ${ }^{1}$ and Yujie Lou ${ }^{1, *}$ \\ ${ }^{1}$ College of Animal Science and Technology, Jilin Agricultural University, Changchun, 130118, China
}

\begin{abstract}
The aim of the present study was to clarify the effect of kisspeptin-10 on LH secretion in prepubertal ewes. In experiment 1, prepubertal ewes fitted with indwelling jugular catheters were randomly assigned to receive $0,0.5,1$ or $2 \mathrm{mg}$ of kisspeptin-10 dissolved in saline, and serial blood samples were collected at 15-min intervals for 180 min to analyze the response curves of LH after injection. In experiment 2, prepubertal ewes fitted with indwelling jugular catheters were injected with 0 or $1 \mathrm{mg}$ of kisspeptin-10 dissolved in saline and the injection was repeated 3 times at $1 \mathrm{~h}$ interval and serial blood samples were collected at 15-min intervals for $210 \mathrm{~min}$ to analyze the response curves of $\mathrm{LH}$ after injection. The results showed that single intravenous administration of $0.5,1$ and $2 \mathrm{mg}$ of kisspeptin-10 all could significantly increased LH secretion in prepubertal ewes, and the effect of 1 and $2 \mathrm{mg}$ of kisspeptin-10 on LH secretion was higher than that of $0.5 \mathrm{mg}$ group. The results also showed that repeated intravenous administration of kisspeptin-10 could effectively increase LH secretion and repeated administration did not influence the effect of kisspeptin-10 on LH secretion in prepubertal ewe. In conclusion, the present study indicated that single or repeated intravenous administration of kisspeptin10 could effectively increase LH secretion in prepubertal ewes. (Key Words: Kisspeptin-10, LH, Prepubertal Ewes)
\end{abstract}

\section{INTRODUCTION}

In mammals, puberty onset is tightly controlled by the hypothalamic-pituitary-gonadal (HPG) axis in which the hypothalamic GnRH plays a crucial role. The hypothalamic $\mathrm{GnRH}$ regulates the secretion of follicle-stimulating hormone (FSH) and luteinizing hormone ( $\mathrm{LH})$ in anterior pituitary gland, which are essential for normal gonadal development and function. Although numerous central players in the inhibitory and stimulatory controls of the GnRH neuron have been identified during the last decade (Ojeda et al., 2003; Terasawa, 2005; Todman et al., 2005), our knowledge of the hypothalamic GnRH neuron regulating reproduction was recently expanded by the identification of KiSS-1/GPR54 system which consists of kisspeptins encoded by KiSS-1 gene and their receptor, G protein-coupled receptor (GPR54) (Ohtaki et al., 2001).

The role of the KiSS-1/GPR54 system in regulating mammalian reproduction was first discovered in late 2003,

\footnotetext{
* Corresponding Author: Yujie Lou.

E-mail: louyujiejlau@126.com

${ }^{2}$ College of Animal Husbandry and Veterinary Medicine, Liaoning Medical University, Jinzhou, 121001, China.

${ }^{3}$ Xuelong Industrial Group, Dalian, 116002, China.

Submitted Oct. 25, 2011; Accepted Jan. 18, 2012; Revised Feb. 5, 2012
}

when two groups independently identified that GPR54 is crucial for the onset of puberty in both men and mice (De Roux et al., 2003; Funes et al., 2003). These findings brought about extensive research on the role of the KiSS1/GPR54 system in reproduction. Detailed pharmacological analyses demonstrated that central and peripheral administration of kisspeptins was able to elicit LH secretion in rodents, monkey, pigs, cattle, sheep, goats and human (Roa et al., 2008; Plant and Ramaswamy, 2009; Hashizume et al., 2010). To our knowledge, although administration of kisspeptins has been shown to elicit the secretion of LH in intact adult sheep (Pompolo et al., 2006) and ovariectomized ewes (Arreguin-Arevalo et al., 2007), there is no data on prepubertal ewes. The present study investigated the effect of single and repeated intravenous administration of kisspeptin-10 on LH secretion in prepubertal ewe.

\section{MATERIALS AND METHODS}

\section{Animals and drugs}

Twelve female Small Tail Han Sheep (native breed), around $16.5 \mathrm{~kg}$ body weigh and three months old were housed in pens, and fed with ad labium access to hay and $150 \mathrm{~g}$ concentrate daily. The ewes were fed twice daily and 


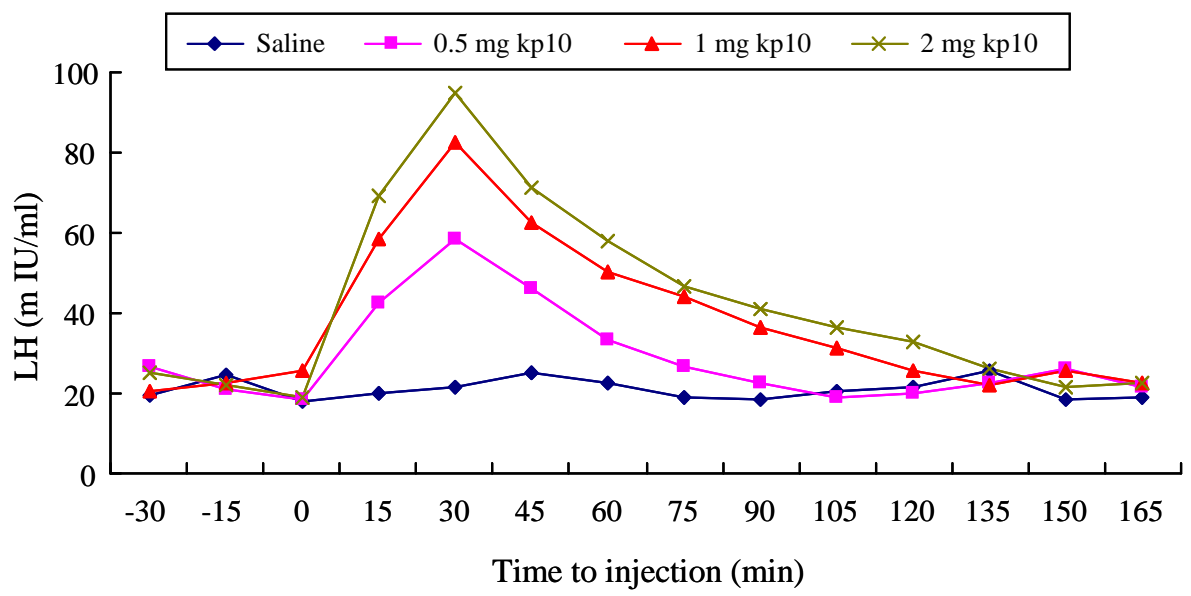

Figure 1. Changes in the serum concentrations of LH over $180 \mathrm{~min}$ in prepubertal ewes that received intravenous injection of $0,0.5,1$ and $2 \mathrm{mg}$ kisspeptin-10 (kp10) dissolved in $5 \mathrm{ml}$ of saline at $0 \mathrm{~min}$.

had free access to water. On the day of the experiment, the ewes were not fed until after the experiment. Ethical approval for the present study was obtained from the Ethical Committee of the Jilin Agricultural University, China. Human kisspeptin-10 amide (amino acid sequence: YNWNSFGLRF-NH $\mathrm{N}_{2}$ ) was synthesized by Shanghai Qiangyao biotechnology Limited.

\section{Experimental designs}

Experiment 1 - Single injection

Twelve female Small Tail Han Sheep were randomly divided into four groups $(n=3)$ and all the ewes were fitted with an indwelling jugular vein catheter twenty-four hours before kisspeptin-10 injection. On the day of the kisspeptin-10 injection, $5 \mathrm{ml}$ of blood samples were collected to obtain serum at 15-min intervals from the ewes for $3 \mathrm{~h}$ (8:00 am to 11:00 am). The ewes were immediately injected with $0,0.5,1$ or $2 \mathrm{mg}$ of kisspeptin-10 dissolved in $5 \mathrm{ml}$ saline respectively after the third sampling. Blood samples were centrifuged at 3,000 rpm for $15 \mathrm{~min}$, and serum was separated and then stored at $-20^{\circ} \mathrm{C}$ for later hormone analysis.

\section{Experiment 2 - Repeated Injection}

Six female Small Tail Han Sheep were divided into two groups $(n=3)$ and prepared in a manner similar to experiment1. On the day of the kisspeptin-10 injection, blood samples were collected to obtain serum at $15-\mathrm{min}$ intervals from the ewes for $210 \mathrm{~min}$ (8:00 am to 11:30 am). After the third sampling, the ewes were injected with 0 or 1 $\mathrm{mg}$ of kisspeptin-10 dissolved in $5 \mathrm{ml}$ saline and the injection was repeated 3 times at $1 \mathrm{~h}$ intervals. Serum was processed and stored as described for experiment 1 .

\section{Hormone analyses}

Serum LH levels were determined using a sheep LH ELISA Kit (Cusabio biotech CO., Ltd). The minimum detectable dose of LH is typically less than $0.12 \mathrm{~m} \mathrm{IU} / \mathrm{ml}$.

\section{Statistical analysis}

To calculate the statistical differences among groups, the statistical package SPSS13.0 (SPSS Incorporated, Chicago) was used for all analysis. The statistical significance of LH concentration (mean and peak) among groups was analyzed by repeated measure ANOVA. In general, $\mathrm{p}$ values less than 0.05 were considered statistically significant.

\section{RESULTS}

Effects of single intravenous injection of different doses of kisspeptin-10 on LH secretion of ewes are shown in Figure 1 and Figure 2. As shown in Figure 1, a single injection of $0.5,1$ and $2 \mathrm{mg}$ of kisspeptin-10 significantly increased the LH secretion compared to injecting with saline within $15 \mathrm{~min}$ of injection. The mean concentration of LH was also increased by injecting $0.5,1$ and $2 \mathrm{mg}$ of

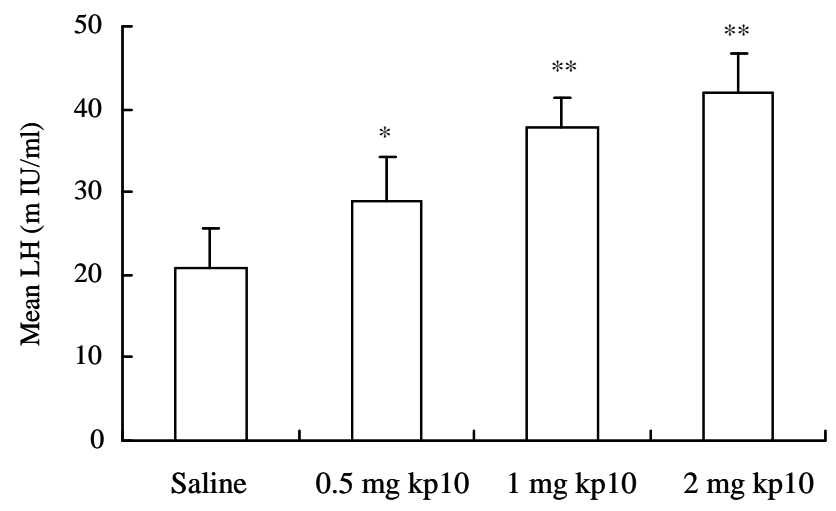

Figure 2. Mean concentration of LH in prepubertal ewes which received intravenous injection of $0,0.5,1$ or $2 \mathrm{mg}$ of kisspeptin-10 (kp10) dissolved in $5 \mathrm{ml}$ of saline at $0 \mathrm{~min} .{ }^{*} \mathrm{p}<0.05, * * \mathrm{p}<0.01$ as compared with that in saline group. 


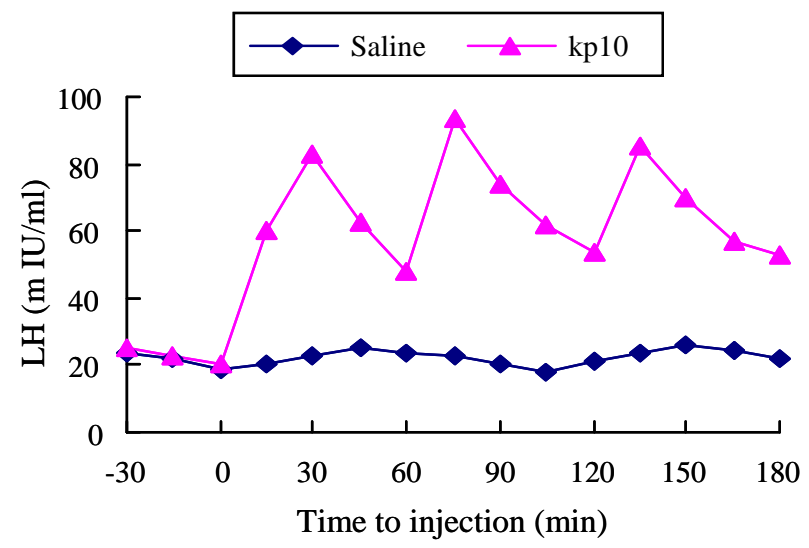

Figure 3. Changes in the serum concentrations of LH over 210 min in prepubertal ewes which received repeated intravenous injection of $1 \mathrm{mg}$ kisspeptin-10 (kp10) dissolved in $5 \mathrm{ml}$ of saline for 3 times at 1-h interval.

kisspeptin-10 (Figure 2). The peak and mean value of LH of ewes treated with $1 \mathrm{mg}$ and $2 \mathrm{mg}$ of kisspeptin-10 were significantly $(p<0.01)$ higher than those of injecting with $0.5 \mathrm{mg}$ of kisspeptin-10, and there was no significant difference between doses of 1 and $2 \mathrm{mg}$. The peak in LH secretion appeared $30 \mathrm{~min}$ after injection of kisspeptin-10. Effect of repeated injection of kisspeptin-10 on LH secretion of ewes is shown in Figure 3. As shown in Figure 3, repeated injection significantly increased the LH secretion $(p<0.01)$ but the effects were not additive.

\section{DISCUSSION}

Although administration of kisspeptin-10 has been shown to elicit the secretion of LH in adult sheep (Pompolo et al., 2006), there are no reports on effect of single or repeated injection of kisspeptin-10 on LH secretion of prepubertal ewes. Results of the present study have demonstrated that a single injection $(0.5,1$, and $2 \mathrm{mg})$ or repeated injection of $1 \mathrm{mg}$ kisspeptin-10 can significantly increase LH secretion of prepubertal ewes.

The effect of kisspeptin-10 on LH secretion may be different with different physiological status. It has been shown that an injection of 1 pmol kisspeptin-10 elicited maximal LH secretion in pubertal but not in juvenile males rats and a higher dose of kisspeptin-10 was needed to stimulate LH secretion in the juvenile rats (Tena-Sempere, 2006). This may be due to the inhibitory state of reproductive axis before puberty which needs more kisspeptin-10 to activate and in vivo experiments showing that the threshold doses of kisspeptins are required to elicit robust LH responses seem to confirm this (Han et al., 2005; Castellano et al., 2006). In ovariectomized adult ewes, intravenous injections of $0.25,1.5$ and $3 \mathrm{mg}$ of kisspeptin10 induced a significant increase in LH secretion, but only half of the ewes were observed to increase $\mathrm{LH}$ concentration in 0.25 and $1.5 \mathrm{mg}$ groups (Arreguin-Arevalo et al., 2007). Very recently, it has been reported that a single intravenous injection of $0.025 \mathrm{mg}, 0.125 \mathrm{mg}$ and $0.25 \mathrm{mg}$ of kisspeptin-10 made adult female goat to increase LH secretion and peak of LH at 20 to 30 min of injection (Hashizume et al., 2010). In addition, time of the effect of kisspeptin-10 on LH secretion of goats lengthened as the injection dose increased (Hashizume et al., 2010).

Results of the present study show that repeated intravenous injection of $1 \mathrm{mg}$ kisspeptin-10 for 3 times at 1 $\mathrm{h}$ interval does increased LH secretion of prepubertal ewes but there was no significant additive effect of additional injections. Similar results were observed in adult goats where three consecutive intravenous injections of $0.25 \mathrm{mg}$ kisspeptin-10 at 2-h intervals also increased LH secretion (Hashizume et al., 2010). In male rats, it was also observed that repeated injections of kisspeptin-10 evoked LH secretion and the degree of which remained constant along the test period (Tovar et al., 2006). These results suggest that kisspeptins may provide a tool for the manipulation of the reproductive axis and the effect of kisspeptins injection on reproduction axis can be affected by breed, physiological stages, and physiological state of animal, injection dose and injection time. Therefore it is necessary to clarify the effect of kisspeptin on reproduction in different condition before kisspeptins was used in regulation of reproduction.

In conclusion, results of the present study suggest that single or repeated intravenous administration of kisspeptin10 could effectively increase LH secretion in prepubertal ewes and the promoting effect might be different in different dose of kisspeptin-10.

\section{ACKNOWLEDGEMENTS}

The work was supported by Educational Department of Liaoning Province (L2010255).

\section{REFERENCES}

Arreguin-Arevalo, J. A., C. A. Lents, T. A. Farmerie, T. M. Nett, and C. M. Clay. 2007. KiSS-1 peptide induces release of LH by a direct effect on the hypothalamus of ovariectomized ewes. Anim. Reprod. Sci. 101:265-275.

Castellano, J., V. Navarro, R. Fernandez-Fernandez, J. Castano, M. Malagon, E. Aguilar, C. Dieguez, P. Magni, L. Pinilla and M. Tena-Sempere. 2006. Ontogeny and mechanisms of action for the stimulatory effect of kisspeptin on gonadotropin-releasing hormone system of the rat. Mol. Cell. Endocrinol. 257:75-83.

De Roux, N., E. Genin, J. Carel, F. Matsuda, J. Chaussain and E. Milgrom. 2003. Hypogonadotropic hypogonadism due to loss of function of the KiSS1-derived peptide receptor GPR54. Proceedings of the National Academy of Sciences of the United States of America. Proc. Natl. Acad. Sci USA. 100:10972-10976.

Funes, S., J. Hedrick, G. Vassileva, L. Markowitz, S. Abbondanzo, 
A. Golovko, S. Yang, F. Monsma and E. Gustafson. 2003. The KiSS-1 receptor GPR54 is essential for the development of the murine reproductive system. Biochem. Biophys. Res. Commun. 312:1357-1363.

Han, S. K., M. L. Gottsch, K. J. Lee, S. M. Popa, J. T. Smith, S. K. Jakawich, D. K. Clifton, R. A. Steiner and A. E. Herbison. 2005. Activation of gonadotropin-releasing hormone neurons by kisspeptin as a neuroendocrine switch for the onset of puberty. J. Neurosci. 25:11349-11356.

Hashizume, T., H. Saito, T. Sawada, T. Yaegashi, A. A. Ezzat, K. Sawai and T. Yamashita. 2010. Characteristics of stimulation of gonadotropin secretion by kisspeptin-10 in female goats. Anim. Reprod. Sci. 118:37-41.

Ohtaki, T., Y. Shintani, S. Honda, H. Matsumoto, A. Hori, K. Kanehashi, Y. Terao, S. Kumano, Y. Takatsu and Y. Masuda. 2001. Metastasis suppressor gene KiSS-1 encodes peptide ligand of a G-protein-coupled receptor. Nature 411:613-617.

Ojeda, S., V. Prevot, S. Heger, A. Lomniczi, B. Dziedzic and A. Mungenast. 2003. Glia-to-neuron signaling and the neuroendocrine control of female puberty. Ann. Med. 35:244255.

Plant, T. M. and S. Ramaswamy. 2009. Kisspeptin and the regulation of the hypothalamic-pituitary-gonadal axis in the rhesus monkey (Macaca mulatta). Peptides 30:67-75.
Pompolo, S., A. Pereira, K. Estrada and I. Clarke. 2006. Colocalization of kisspeptin and gonadotropin-releasing hormone in the ovine brain. Endocrinology 147:804-810.

Roa, J., E. Aguilar, C. Dieguez, L. Pinilla and M. Tena-Sempere. 2008. New frontiers in kisspeptin/GPR54 physiology as fundamental gatekeepers of reproductive function. Front. Neuroendocrinol. 29:48-69.

Tena-Sempere, M. 2006. GPR54 and kisspeptin in reproduction. Hum. Reprod. Update 12:631-639.

Terasawa, E. 2005. Role of GABA in the mechanism of the onset of puberty in non-human primates. Int. Rev. Neurobiol. 71:113-129.

Todman, M., S. Han and A. Herbison. 2005. Profiling neurotransmitter receptor expression in mouse gonadotropinreleasing hormone neurons using green fluorescent proteinpromoter transgenics and microarrays. Neuroscience 132:703712.

Tovar, S., M. J. Vázquez, V. M. Navarro, R. Fernández-Fernández, J. M. Castellano, E. Vigo, J. Roa, E. F. Casanueva, E. Aguilar, L. Pinilla, C. Dieguez and M. Tena-Sempere. 2006. Effects of single or repeated intravenous administration of kisspeptin upon dynamic LH secretion in conscious male rats. Endocrinology 147:2696-2704. 